\title{
Future rapid appraisal work of the IDS women's cluster
}

\section{Kate Young}

One of the underlying reasons for organising the two workshop meetings on rapid appraisal was our concern with the development of a more adequate metholodology for feminist research. Members of the women's cluster have been considering this problem for some time and have recently been investigating ways in which training in research in to development issues from a feminist perspective might be carried out. There are several reasons for the need for such a programme.

In the past ten years or so an enormous amount of research has been undertaken into issues of what is generally called women and development. A great deal of the literature this has generated has been valuable in that it has begun to fill many of the gaps in our knowledge about what women in the underdeveloped countries do (just as feminists have provided contemporary and historical data on women's lives in the so-called developed countries). It has pointed to the economic importance of women's work, its centrality to the strategies of survival of family and household units. Some of this material has also undermined a number of commonly held assumptions - such as that of equal sharing or pooling within the family (an assumption which used to pervade development thinking but which is less confidently made today); that of the substitutability of labour in agricultural tasks (the degree of rigidity of the sexual division of labour differs widely from society to society, but there is none without it); that of women's lesser work input and their lower productivity (in some cases women work longer hours than men, even discounting domestic duties; in many tasks they are appreciably more productive). It has pointed to a number of neglected issues - for example, that of the growth of women-headed households in both rural and urban areas (although the underlying reasons are different); inter-familial violence and the defencelessness in particular of older and widowed women.

However, much of this literature is very descriptive. Much of it is rather partial - it gives a picture of what women do but not how this fits into the totality of social production, nor how women and men think about what they do and what their sensitivity to change may be. Much of it is rather functional in terms of explanations: women do $\mathrm{x}$ because it benefits capital, or the process of profit-making, or their husbands' kin-group. There is little sense of tension and contradiction. Much of the analysis is also rather static; it is rarely made clear how the society being described or the sets of organisational practices discussed are changing, or in what direction, to benefit whom. To some extent this rather narrow and static vision is produced by the concentration on women and their roles, rather than on relations between men and women, the content of such relationships, and the meanings given them by both women and men. For this reason the Subordination of Women cluster ${ }^{1}$ has always insisted on the basic principle that our object of study is not women but socially constituted relations between men and women (the social relations of gender).

Gender as a term has been widely adopted into social science terminology although it is sometimes used as a simple alternative to the term sex. Gendering refers to the complex process by which males and females become socially men and women who bear within themselves, in the meanings they attach to their own actions, desires and projects, the imprint of sociallyorganised directives as to what constitutes masculinity and femininity. The term gender then refers to the product of this social organisation of the sexes into two distinct and different categories: men and women. Relations between women and men are thus not spontaneous but socially defined and shaped. People are, of course, not only gendered, but also allocated to certain kinship positions, social strata, or classes. The effect of this is that neither the category men nor that

\footnotetext{
1 This is an abbreviation of the full but unwieldy title: the Continuing Subordination of Women in the Development Process.
}

WDS Bulletin. 1984, vol 15 no 1. Institute of Development Studies. Sussex 
of women is homogeneous. An implication is that it is difficult to suggest that at all times in a given culture and economy the interests of all men (or of all women) are identical.

As we noted in our first Bulletin on the work of the Subordination of Women collective, women (or men) is an empirical term, not an analytical one [Bulletin vol 10 no 3, April 1979, p10]. What has to be investigated is how relations between the genders are transformed, reinforced or reshaped during the process of economic development to the detriment or benefit of which sector of society. Only when this is understood can steps be taken to modify detrimental tendencies so that gender equality becomes an effect of economic development and a premise for true social development.

The difficulty of such an approach is that it considerably complicates the research process. It is not possible to collect relational data by means of simple questionnaires applied by a number of paid assistants, rather the researcher has to gain the confidence of the respondents. To get some idea of process and of the direction of change lengthier research methods are also needed (life histories, case histories, other historical data). Yet this poses a critical problem: that of the time it takes to do in-depth qualitative research and - possibly more crucially - its cost. The type of lengthy field study which takes a year or so to collect the data and anything up to a decade to analyse and publish the findings is probably increasingly unrealistic as a research method. In many underdeveloped countries research budgets are extremely slender, research funds from international agencies are shrinking, and in Britain at least social science research funding has diminished notably in recent years. So it has become of increasing urgency to develop ways of generating sufficiently detailed and contextualised data at least cost.

Much of our discussions during the workshops centred on what sort of data can reasonably be collected by means of rapid appraisals, and how they are best organised. One of the clearest organisational points made was that rapid appraisal only refers to the period of actual fieldwork; in order to conduct a successful rapid appraisal, preliminary work has to be both painstaking and detailed (and thus timeconsuming). That is, all available data on the area or topic to be researched have to be brought together for intensive study; the researcher must not only be able to identify the gaps in the data and its inconsistencies, but also must have some idea of what interconnections to look for in the field.

If, for example, existing work patterns are the principal object of the research (perhaps because a potential development project will disrupt or build itself around them) then a number of hypotheses should be made as to what they in turn may be related to, so as to understand how change in work patterns may affect other social arrangements in the society. Hypothesis building is a useful tool because it can stimulate researchers to try to deduce from their rapidly developing knowledge of the area what interconnections are possible. Hypothesis making and testing can provide a focus for the preliminary research. This is possible not only where some knowledge of an area already exists, but even in a relatively unknown area which is culturally similar to a known one, although economically different.

Another point made by a number of participants was the use of local people as research partners. Local people (not only in terms of being from the country but also those who actually live in the fieldwork area) are an enormous source of information, and when brought into a project and fully informed as to its aims can be of invaluable assistance.

The question of training is crucial, particularly as far as expanding work in the area of gender and social transformation is concerned. In most countries the number of people with research experience in gender issues is rather small, although frequently there are often very many willing apprentices. The IDS women's cluster, in response to a number of requests, has for the past couple of years been thinking about ways to train grassroots researchers, and indeed the discussions at the workshop were an important aspect of the further development of our ideas. So far the cluster has been able to prepare a preliminary draft of a teaching manual to be used in a workshop context to discuss some of the research issues around rural development and gender relations. It is anticipated that in the course of the workshop, participants will convert the teaching manual into a research tool by rewriting it to suit their own needs, by incorporating into it their own specialised knowledge of their own society. So far the manual is directed to rural research and covers three main themes: work, fertility and consciousness. At a future stage, it is hoped to extend it to include themes relevant to urban research, including formal and informal employment, health, housing, and political participation. 